\title{
Functional Laparoscopic Roux-en-Y Gastric Bypass with Fundectomy and Gastric Remnant Exploration (LRYGBfse)—a Video Vignette
}

\author{
Giovanni Lesti ${ }^{1}$ - Marco Antonio Zappa ${ }^{2} \cdot$ Francesco Lesti $^{1} \cdot$ Davide Bona $^{3} \cdot$ Alberto Aiolfi $^{3}$ (D) \\ Received: 22 November 2020 / Revised: 10 February 2021 / Accepted: 10 February 2021 / Published online: 22 February 2021 \\ (C) The Author(s) 2021
}

\begin{abstract}
Background The laparoscopic Roux en-Y gastric bypass (LRYGB) is performed worldwide and is considered by many the gold standard treatment for morbid obesity. However, the difficult access to the gastric remnant and duodenum represents intrinsic limitations. The functional laparoscopic gastric bypass with fundectomy and gastric remnant exploration (LRYGBfse) is a new technique described in attempt to overcome the limitations of the LRYGB. The purpose of this video was to demonstrate the LRYGBfse in a 48-year-old man with type II diabetes and hypertension.

Methods An intraoperative video has been anonymized and edited to demonstrate the feasibility of LRYGBfse.

Results The operation started with the opening of the gastrocolic ligament. Staying close to the gastric wall, the stomach is prepared up to the angle of His. After the placement of a 36-Fr orogastric probe, gastric fundectomy is completed in order to create a $30 \mathrm{cc}$ gastric pouch. A polytetrafluoroethylene banding (ePTFE) is placed at the gastro-gastric communication, $7 \mathrm{~cm}$ below the cardia, and gently closed after bougie retraction. The bypass is completed by the creation of an antecolic Roux-en-Y $150 \mathrm{~cm}$ alimentary and $150 \mathrm{~cm}$ biliopancreatic limb.

Conclusion The LRYGBfse is a feasible and safe technique. The possibility to endoscopically explore the excluded stomach with an easy access to the Vater's papilla is a major advantage. Further studies are warranted to deeply explore and compare outcomes with the standard LRYGB.
\end{abstract}

Keywords Laparoscopic functional gastric bypass · Bariatric surgery $\cdot$ Gastric bypass · Video vignette

\section{Introduction}

The laparoscopic Roux en-Y gastric bypass (LRYGB) is performed worldwide and is considered by many the gold standard treatment because of its excellent results in term of weight loss, comorbid resolution, and quality of life improvement $[1,2]$. However, the challenging diagnosis and treatment of developing diseases in the gastric remnant, duodenum, and common bile duct are limits [3]. The functional laparoscopic

Alberto Aiolfi

alberto.aiolfi86@gmail.com

1 Department of General Surgery, Fondazione Salus Clinica Di Lorenzo, Via Vittorio Veneto 37, Avezzano, AQ, Italy

2 Department of General and Emergency Surgery, Ospedale Fatebenefratelli Sacra Famiglia, University of Milan, Milan, Italy

3 Department of Biomedical Science for Health, Division of General Surgery, Istitituto Clinico Sant'Ambrogio, University of Milan, Milan, Italy gastric bypass with fundectomy and gastric remnant exploration (LRYGBfse) is a relatively new technique introduced in attempt to overcome the limitations of the LRYGB with promising preliminary results [4].

\section{Purpose}

The purpose of this video was to demonstrate the fashioning of a LRYGBfse in a 48-year-old morbidly obese man (weight $147 \mathrm{~kg}$; BMI $42.8 \mathrm{~kg} / \mathrm{m}^{2}$ ) with hypertension and type II diabetes.

\section{Methods}

An intraoperative video has been edited to demonstrate the feasibility of the LRYGBfse. Written informed consent was obtained from the patient. 


\section{Results}

The operation started with the opening of the gastrocolic ligament. Staying close to the gastric wall, the stomach that is prepared up to the angle of His and care is taken to seal the short vessels and to free any posterior adhesions. The left pillar dissection is performed meticulously and its fibers need to be exposed. After the placement of a 36-Fr orogastric probe, the gastric fundectomy is completed with sequential Endo GIA $^{\mathrm{TM}}$ linear stapler firings (Medtronic, Minneapolis, MN, USA) in order to create a $30 \mathrm{cc}$ gastric pouch. A polytetrafluoroethylene banding (ePTFE) is placed at the gastro-gastric communication $(7 \mathrm{~cm}$ below the cardia) and gently closed after bougie retraction. The bypass is completed by the creation of an antecolic Roux-en-Y $150 \mathrm{~cm}$ alimentary and $150 \mathrm{~cm}$ biliopancreatic limb. The common channel is measured about $300 \mathrm{~cm}$. In case the common channel is shorter than $300 \mathrm{~cm}$, the alimentary limb is shortened. Linear side-toside gastrojejunal and jejuno-ileal anastomosis (30-mm Endo GIA $^{\mathrm{TM}}$ ) are fashioned. The Petersen's defect is closed with absorbable sutures.

\section{Discussion}

The LRYGBfse has been described in attempt to overcome the limitations of the LRYGB with encouraging preliminary results $[3,4]$. The creation of a gastro-gastric virtual communication is the result of a specific and well-calibrated surgical procedure. The ePTFE band gently closes the gastro-gastric communication, and the bolus is diverted through the alimentary limb with duodenal and jejunal functional exclusion. As documented by the postoperative gastrographin swallow study, the radiopaque bolus is rapidly diverted through the gastrojejunal anastomosis and the alimentary limb. The prompt arrival of bolus in the foregut and hindgut results in an increased glucagon-like peptide 1 (GLP-1) and peptide YY (PYY) secretion [5].

The resection of the gastric fundus is performed for hormonal, physiological, and technical reasons with a significantly reduced secretion of ghrelin and consequent effect on glucose homeostasis and appetite [6]. These findings suggest that the fundus resection with concomitant ghrelin suppression could be an effective adjunct in the treatment of type II diabetes [7].

Notably, the passage of 9-mm and 13-mm endoscopes for diagnostic and therapeutic purposes is allowed towards the gastro-gastric communication by gentle pushing (Fig. 1) [4]. Systematic endoscopic exploration of the gastric remnant and duodenum are performed yearly during follow-up. Endoscopic retrograde cholangiopancreatography with sphincterotomy was necessary in two patients for symptomatic choledocholithiasis, and another patient was diagnosed with prepyloric cancer 15 months after the index procedure. The

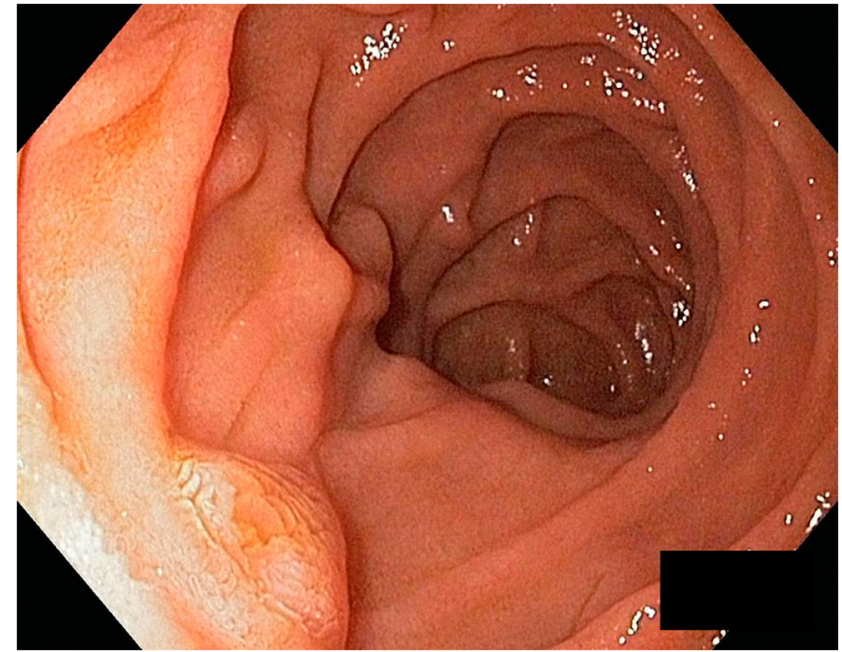

Fig. 1 The duodenum and the Vater's papilla may be reached and explored with 9-mm and 13-mm endoscopes for diagnostic and therapeutic purposes

feasible exploration of the remnant remains a critical issue considering the increasing number of LRYGB performed each year worldwide in a population of young patients that will probably need a screening evaluation in the future $[8,9]$.

\section{Conclusion}

The LRYGBfse is a new feasible and safe technique. The possibility to endoscopically explore the excluded stomach with an easy access to the Vater's papilla is a major advantage. Further studies are warranted to deeply explore and compare outcomes with the standard LRYGB.

Supplementary Information The online version contains supplementary material available at https://doi.org/10.1007/s11695-021-05298-w.

Funding Open access funding provided by Università degli Studi di Milano within the CRUI-CARE Agreement.

\section{Declarations}

Informed Consent Written informed consent was obtained from the individual participant included in this video.

Conflict of Interest The authors declare no competing interests.

Human and Animal Rights All procedures performed in studies involving human participants were in accordance with the ethical standards of the institutional and/or national research committee and with the 1964 Helsinki declaration and its later amendments or comparable ethical standards. 
Open Access This article is licensed under a Creative Commons Attribution 4.0 International License, which permits use, sharing, adaptation, distribution and reproduction in any medium or format, as long as you give appropriate credit to the original author(s) and the source, provide a link to the Creative Commons licence, and indicate if changes were made. The images or other third party material in this article are included in the article's Creative Commons licence, unless indicated otherwise in a credit line to the material. If material is not included in the article's Creative Commons licence and your intended use is not permitted by statutory regulation or exceeds the permitted use, you will need to obtain permission directly from the copyright holder. To view a copy of this licence, visit http://creativecommons.org/licenses/by/4.0/.

\section{References}

1. Angrisani L, Santonicola A, Iovino P, et al. Bariatric surgery worldwide. 2013. Obes Surg. 2015;25(10):1822-32.

2. Buchwald H, Oien DM. Metabolic/bariatric surgery worldwide. 2011. Obes Surg. 2013;23:427-36.

3. Lesti G, Aiolfi A, Mozzi E, et al. Laparoscopic gastric bypass with fundectomy and gastric remnant exploration (LRYGBfse): results at 5-year follow-up. Obes Surg. 2018;28(9):2626-33.

4. Lesti G, Bona D, Sozzi A, et al. Impact of functional laparoscopic gastric bypass with fundectomy and gastric remnant exploration
(LRYGBfse) on patients' quality of life: trajectory and 5-year follow-up result. Obes Surg. 2020;30(8):3046-53.

5. Chronaiou A, Tsoli M, Kehagias I, et al. Lower ghrelin levels and exaggerated postprandial peptide-YY, glucagon-like peptide-1, and insulin responses, after gastric fundus resection, in patients undergoing Roux-en-Y gastric bypass: a randomized clinical trial. Obes Surg. 2012;22(11):1761-70.

6. Christou NV, Look D, Maclean LD. Pre-and post-prandial ghrelin levels do not related with satiety or failure to achieve a successful outcome after Roux-en-Y gastric bypass. Obes Surg. 2005;15:101723.

7. Porta A, Aiolfi A, Musolino C, et al. Prospective comparison and quality of life for single-incision and conventional laparoscopic sleeve gastrectomy in a series of morbidly obese patients. Obes Surg. 2017;27(3):681-7.

8. Aiolfi A, Asti E, Rausa E, et al. Trans-Gastric ERCP After Roux-enY gastric bypass: systematic review and meta-analysis. Obes Surg. 2018;28(9):2836-43.

9. Tornese S, Aiolfi A, Bonitta G, et al. Remnant gastric cancer after Roux-en-Y gastric bypass: narrative review of the literature. Obes Surg. 2019;29(8):2609-13.

Publisher's Note Springer Nature remains neutral with regard to jurisdictional claims in published maps and institutional affiliations. 